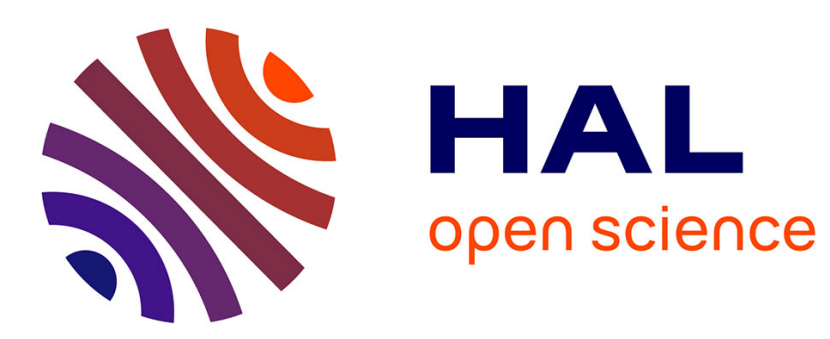

\title{
Notes forestières sur l'Ouest de l'U.R.S.S. de Léningrad à la Georgie
}

\author{
Jean de Vaissiere, Jean Pourtet, Paul Turpin, Yves Chevalier
}

\section{To cite this version:}

Jean de Vaissiere, Jean Pourtet, Paul Turpin, Yves Chevalier. Notes forestières sur l'Ouest de l'U.R.S.S. de Léningrad à la Georgie. Revue forestière française, 1960, 6, pp.382-407. $10.4267 / 2042 / 24286$. hal-03389702

\section{HAL Id: hal-03389702 \\ https://hal.science/hal-03389702}

Submitted on 21 Oct 2021

HAL is a multi-disciplinary open access archive for the deposit and dissemination of scientific research documents, whether they are published or not. The documents may come from teaching and research institutions in France or abroad, or from public or private research centers.
L'archive ouverte pluridisciplinaire HAL, est destinée au dépôt et à la diffusion de documents scientifiques de niveau recherche, publiés ou non, émanant des établissements d'enseignement et de recherche français ou étrangers, des laboratoires publics ou privés. 


\section{NOTES FORESTIĒRES SUR L'OUEST DE L'U.R.S.S. DE LENINGRAD A LA GEORGIE}

Dans le cadre des relations culturelles et dans le but de faciliter les contacts et les échanges de vues entre techniciens français et soviétiques, le Gouvernement de l'Union Soviétique a invité 4 représentants de l'Administration des Eaux et Forêts à participer à un voyage d'études de 5 semaines en U.R.S.S. à l'automne 1959.

La délégation française (1) a visité du 16 septembre au 20 octobre 1959 la région de Moscou, l'Ukraine y compris la Crimée, la Géorgie et pour terminer Léningrad et ses environs. Au total près de $10000 \mathrm{~km}$ ont été parcourus, en avion, en chemin de fer, en bateau et en voiture automobile, sans compter bien entendu l'aller et retour Paris-Moscou (6000 km environ).

Ces déplacements se sont effectués entre le $60^{\circ}$ degré de latitude Nord (Léningradl) et le 41e degré (Tbilisi), c'est-à-dire de la latitude d'Helsinki à celle de Barcelone. En longitude, le déplacement n'a été que de $17^{\circ}$ (du $28^{\mathrm{e}}$ au $45^{\mathrm{e}}$ (legré Est) mais la France occupe moins de $13^{\circ}$ en longitude de la Pointe du Raz à Wissembourg... A notre échelle. ce périple était clonc d'une ampleur indiscutable, à l'échelle du véritable continent que représente l'U.R.S.S., il nous a seulement permis d'effleurer sa bordure occidentale et comme le montre une carte forestière de l'Union, il s'est effectué en dehors de la grande zone forestière soviétique qui approvisionne le pays en bois. Cela avait l'avantage de nous faire visiter des régions relativement proches écologiquement et forestièrement de notre pays mais explique, comme nous le verrons plus loin, que dans les régions parcourues les forêts jouent un rôle touristique, de protection et sanitaire plutôt que de production. Nous ne parlerons donc pas de cette dernière, ni surtout de l'exploitation forestière à laquelle $M$. le

(1) Elle était composée de :

MM. J. DE VAISsière, Inspecteur Général des Eaux et Forêts, chef de la Délégation,

J. Pourtet, Conservateur des Eaux et Forêts,

P. Turpin, Ingénieur des Eaux et Forêts,

Y. Chevalier, Ingénieur des Eaux et Forêts.

Le présent article est leur œuvre collective. 
Conservateur Venet a consacré il y a deux ans un article ici même (2).

Avant de passer à l'étude du milieu et des problèmes forestiers rencontrés au cours du voyage, quelques mots nous semblent néces-

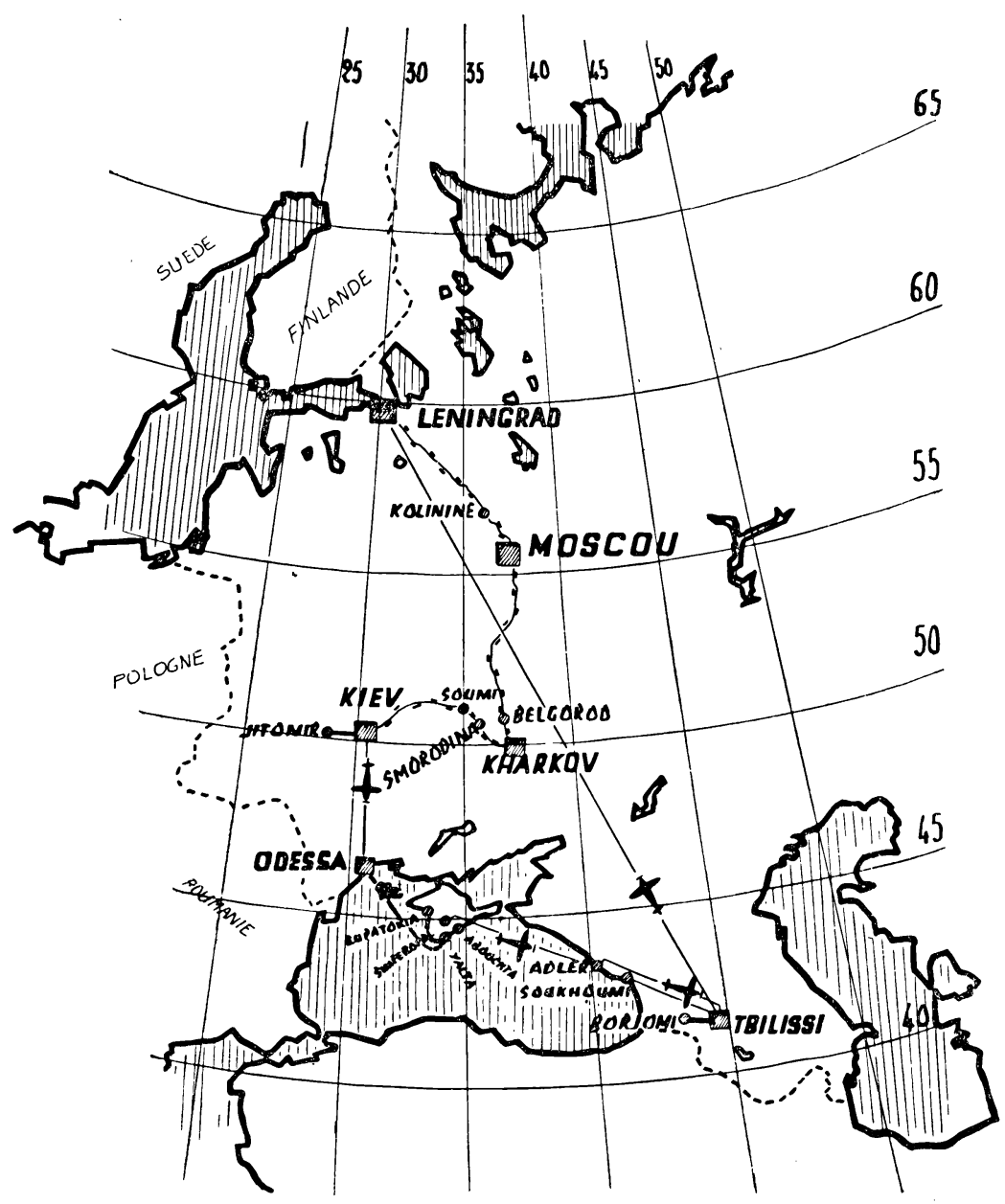

saires sur l'organisation administrative et politique de l'U.R.S.S., fédération de 15 républiques ayant chacune leurs particularités propres: nous avons partiellement visité trois d'entre elles: les régions

(2) J. VeNET. - Quelques impressions sur les exploitations forestières en U.R.S.S. Revue Forestière Française, tome X, 1958, p. 467-480. 
de Moscou et de Léningrad dans l'immense R.S.F.S.R. qui comprend la Russie proprement dite... mais s'étend aussi sur la plus grande partie de la Sibérie jusqu'à Vladivostok; la République d'Ukraine que nous avons traversée du Nord au Sud, enfin au delà du Caucase la République de Géorgie aux portes de 1'Asie: elle est bilingue et la langue nationale comporte un alphabet complètement différent de l'alphabet cyrillique.

Les diffórences climatiques entrainées par les différences de latitude, si elles sont considérables, ne sont pas homologues à celles qu'on constaterait en Europe occidentale soumise dans son ensemble à un régime océanique, la Russie est au contraire soumise à un climat continental fortement influencé par l'énorme masse des terres d'Asie. La proximité de mers plus ou moins fermées au nord (golfe de Finlande - Baltique) et au Sud (Mer Noire) atténue à leurs abords le caractère continental mais ne le fait pas disparaître, surtout en hiver: les isothermes de janvier forment des racs de cercle à grand rayon ayant leur centre en Sibérie et sensiblement orientés nord-sud en Russie d'Europe. Aussi le frois hivernal est-il d'autant plus marqué qu'on se déplace de l'ouest vers l'est et non du sud vers le Nord.

La température moyenne de janvier est basse partout: elle est la même à Léningrad ( $60^{\circ}$ de latitude nord) et à Kharkov, $10^{\circ}$ de latitude plus au sud mais à l'intérieur des terres et elle diffère peu entre Odessa et Riga!

C'est seulement au sud de la chaîne du Caucase et de son prolongement occidental en Crimée que les moyennes de janvier sont positives (rappelons qu'en France, il n'y a pas de moyenne de janvier négative en (lehors des montagnes).

En été, par contre, les températures moyennes sont élevées partout ( $+17^{\circ} 5$ à Léningrad pour juillet) et les isothermes de juillet sont sensiblement sud-ouest - nord-est, la moyenne atteignant $24^{\circ}$ sur la côte de la Mer Noire.

Ces divers chiffres sont d'autant plus faciles à comparer que les altitudes sont très basses et uniformes, de faibles bombements rompant rarement la plaine russe de Léningrad aux abords de la Crimée. Il n'en est plus de même en Crimée et en Georgie où les. fortes variations d'altitudes déterminent des modifications corrélatives de climat.

Sauf pour ces montagnes, la pluviosité est peu élevée ou même faible: dans les régions visitées, elle va d'à peine $600 \mathrm{~mm}$ à Jitomir à $400 \mathrm{~m}$ m seulement à Odessa et elle n'est que de $500 \mathrm{~mm}$ à Moscou. Cependant, partout le nombre de jours de pluie est relativement grand, compte tenu de ces chiffres et surtout la nébulosité et l'état hygrométrique sont presque constamment élevés. 
En outre, la neige couvre longtemps le so1: 140 jours à Léningrad, 146 à Moscou, 20 à 40 seulement à Odessa.

Les sols semblent beaucoup plus liés aux conditions climatiques et à la végétation qu'à la roche-mère: il nous a d'ailleurs été difficile de connaitre le substratum géologique des terrains parcourus: les Russes, fondateurs de la pédologie, attachent beaucoup plus d'importance à celle-ci qu'à la géologie. Si c'est compréhensible sur les profonds sols de loess d'Ukraine, cela nous a paru moins logique sur les sols sur glaciaire ou sur roches anciennes de la région de Léningrad et à fortiori pour ceux qui reposent sur les formations géologiques variées des régions montagneuses.

Au point de vue végétation, 3 types s'étagent du nord au sud: la taïga, forêt boréale, passant à la forêt mixte feuillus-résineux de Léningrad-Moscou, la forêt feuillue du nord-ouest de l'Ukraine, s'entr'ouvrant progressivement au niveau de Kiev pour faire place au sud à la steppe sans arbre. On distingue une zone intermédiaire dite " steppe boisée ), probablement d'origine anthropogène, là où les conditions climatiques rendent précaire la solidité de la forêt.

Dans les montagnes méridionales, on trouve à la base les formations classiques de steppes, de maquis et de garrigues, passant aux formations forestières feuillues et résineuses des montagnes, puis s'éclaircissant aux hautes altitudes du Caucase pour faire place aux prairies alpines (vers $2500 \mathrm{~m}$ ).

\section{Organisation du Service de gestion, de l'enseignement et des recherches forestières}

Rappelons d'abord qu'il existe des échelons fédéraux (Office Central au Ministère de l'Agriculture de l'U.R.S.S. à Moscou, Institut National de recherches scientifiques sur la Sylviculture et la mécanisation des travaux forestiers à Pouchkino près de Moscou, Académie des sciences agricoles de l'U.R.S.S.) qui ont un rôle de coordination entre les organismes similaires et généralement plus étoffés des républiques.

Nous allons examiner leur fonctionnement dans la République d'Ukraine: à cela plusieurs raisons, nous nous y sommes attardés assez longuement, avons pu avoir de fructueuses discussions avec les fonctionnaires forestiers et surtout l'Ukraine est à notre échelle - 42 millions d'habitants, $600000 \mathrm{~km}^{2}, 8$ millions d'hectares de forêts - et à notre latitude (entre les $43^{\circ}$ et $52^{\circ} \mathrm{L} \mathrm{N}$ ). Son taux de boisement est plus faible que celui de la France - $12 \%$ mais la steppe qui occupe une importante partie au sud-est du pays requiert l'activité des forestiers. Quant aux formations forestières, elles rappellent les nôtres, chêne pédonculé et pin sylvestre sont les principales essences ukrainiennes et on va de la forêt sapin-hêtre- 


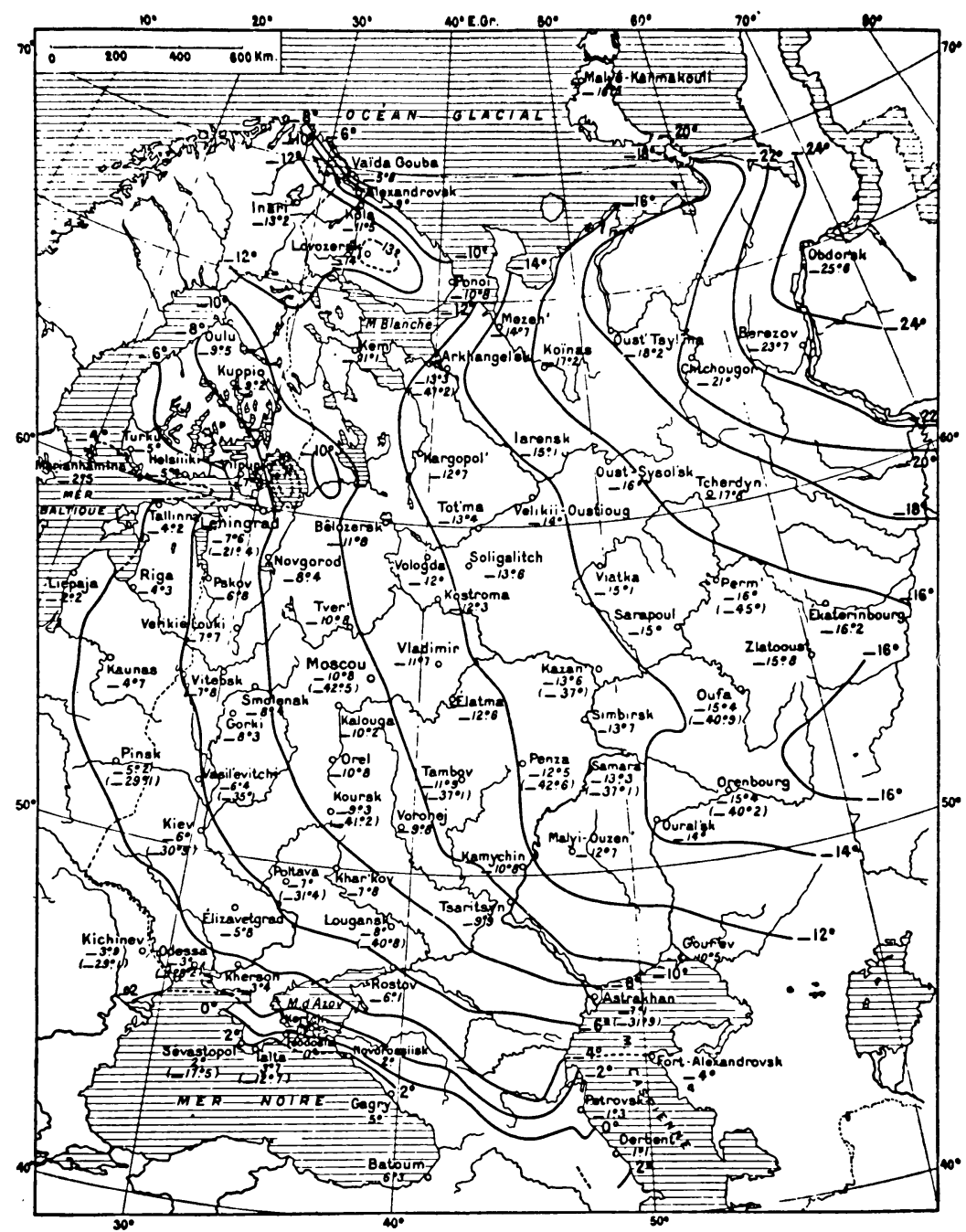

Isothermes de janvier.

Principalement d'après l'Atlas de l'Observatoire central de Géophysique, Livr. I, Leningrad, 1927. - Les lignes isothermes ont été tracées en supposant les altitudes uniformément réduites à 0 mètre. Les chiffres donnent les températures moyennes calculées sans correction d'altitudes. Les chiffres entre parenthèses indiquent les minima enregistrés. 


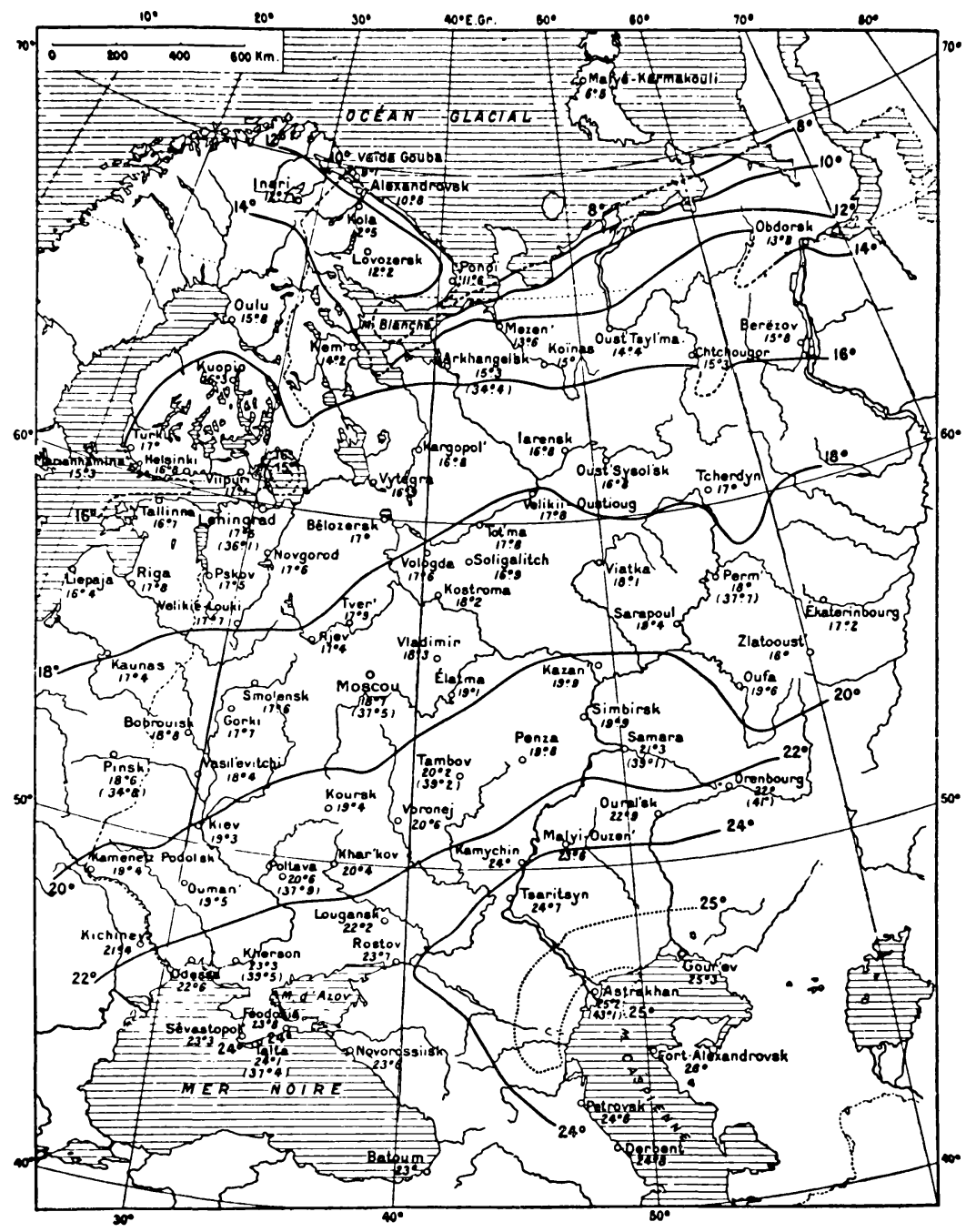

Isothermes de juillet.

Principalement d'après l'Atlas de l'Observatoire central de Géophysique, Livr. I, Leningrad, 1927. - Les lignes isothermes ont été tracées en supposant les altitudes uniformément réduites à 0 mètre. Les chiffres donnent les températures moyennes calculées sans correction d'altitudes. Les chiffres entre parenthèses indiquent les maxima enregistrés. 
épicéa dans les montagnes de l'Ouest au maquis méditerranéen en Crimée.

Les exploitations forestières industrielles ne sont pas à la charge du Service forestier qui s'occupe de la gestion proprement dite et des travaux en forêt ainsi que de la chasse. Les principales forêts sont les forêts d'Etat, mais il existe aussi des forêts kolkhoziennes assez comparables à nos forêts communales, également gérées par le Service de la République mais avec contrôle d'un Comité Directeur élu.

Voici, schématiquement, l'organisation du service forestier ukrainien:

\section{Directeur Général}

Chef

du Département de Sylviculture

et des Bandes forestières protectrices

au Ministère d'Agriculture de l'Ukraine à Kiev

25 Directions régionales de la Sylviculture

avec à leur tête un Forestier Général assisté

d'un Directeur Administratif disposant de 2 bureaux

(comptabilité et plan économique - autofinancement)

et d'un Ingénieur Principal technique disposant de 5 bureaux dirigés par des Ingénieurs (Gestion-Reboisements-Protection des Forêts - Forêts kolkhoziennes-Chasse).

Chaque direction régionale comprend un certain nombre de leskhoz ou inspection (jusqu'à 17)

Leskhoz (10000 à 30000 ha de forêts)

avec 1 Ingénieur chef cle service, son adjoint, 4 ingénieurs et 6 employés

Le chef du leskhoz dispose d'une voiture tout terrain " Gaz ».

Lesnitchestvo (Cantonnement: 4 à 8 par Leskhoz)

avec 1 Ingénieur assisté d'un technicien et d'un comptable

Obiezl (District: 2 à 4 par Cantonnement)

avec 1 Technicien

Obkhod (Triage: 2 à 6 par district)

avec 1 liesnik

Le liesnik ou " agent technique » dirige les ouvriers, il loge en maison forestière: c'est souvent un praticien mais ce devrait être un " technicien » (il peut accéder à ce niveau grâce à un enseignement par correspondance).

L'organisation est assez proche de la nôtre, mais les cantonnements ont une surface de forêts comparable à celle de nos districts.

L'originalité de lorganisation financière du service forestier consiste dans l'affectation du produit des aoupes non industrielles 


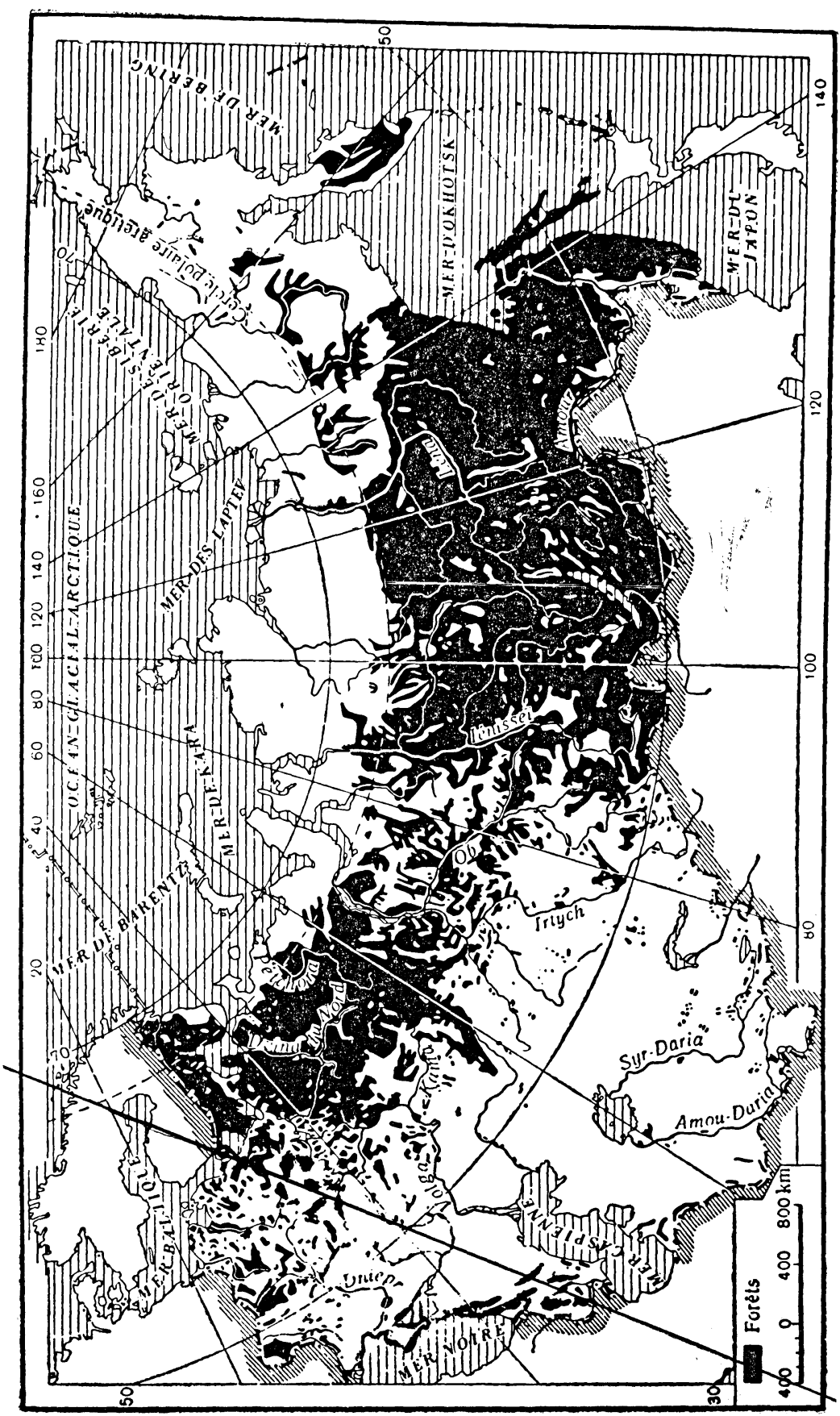


(éclaircies, coupes sanitaires, menus produits, etc...) à un bureau d'autofinancement assurant la création et le fonctionnement de petites industries et pouvant réaliser certains travaux (routes, ponceaux, etc...).

La Chasse appartient à une Société régionale de chasse, subdivisée en sociétés de district: une cotisation annuelle de 36 roubles (18 NF environ) donne le droit de chasse réglementé du point de vue période. Les animaux nuisibles en particulier les loups et les renards peuvent être détruits toute l'année.

Nous avons employé les expressions d’ingénieurs, de techniciens et de praticiens: elles correspondent pratiquement aux trois types d'enseignement, supérieur, secondaire ou technique et " d'instruction générale ». Ce dernier est assuré par l'école unique obligatoire penclant 7 ans de 7 à 14 ans. C'est à cet âge que les jeunes gens désirant devenir ( technicien ) peuvent entrer, après concours, dans les “Technicum ) ou écoles professionnelles: celle que nous avons visitée clans les environs de Kharkov à Tchougouiev-Babka et où nous avons été très aimablement reçus comprenait 570 élèves réguliers filles et garçons répartis en 3 sections, celle de sylviculture (30\% de filles) formant surtout des “ Obiezd ) (équivalent de nos chefs de district), celle de mécanique agricole formant les chefs cl'équipe des kolkhozes, enfin celle cle comptabilité essentiellement féminine et où les études clurent moins de 2 ans au lieu de 4 pour les autres. Un enseignement par correspondance avec stage à l'Ecole pendant les congés payés double pratiquement le nombre des élèves. L'Ecole dispose d'un vaste domaine forestier et agricole de 13500 ha et ses ateliers en particulier sont fort bien équipés.

L’instruction générale peut être poussée jusqu'à 17 ans, en particulier pour l'accès aux Ecoles supéricures: celles qui forment des Ingénieurs forestiers sont appelées Académies Agricoles et dépendent du Ministère de l'Agriculture, elles sont au nombre de 3 pour l'Ukraine: Kharkov, Lvow et Kiev. Nous avons visité l'Académie Agricole de Kiev, admirablement située sur une colline de la banlieue de la capitale ukrainienne au milieu d'un domaine boisé de 22000 ha. Elle dispose en outre d'une autre forêt parc et d'un arboretum de 6 ha. Elle comprend 10 facultés formant les spécialistes cles cliverses branches de la technique agricole:

Agronomie

Agrochimie

Zootechnie

Vétérinaire

Mécanisation Agricole
Electrification agricole

Exploitation forestière

Industrie du bois

Sylviculture

Economie rurale 
Le recrutement des étudiants se fait par concours (5 à 10 candiclats pour une place) parmi les élèves ayant terminé leur cycle d'instruction générale de 10 années et ayant accompli si possible 2 ans de pratique: ils sont âgés de 18 à 35 ans à l'entrée, mais le plus couramment ont 20 ans et sortent à 25 ans.

Chaque année, 1400 étudiants entrent à l'Académie qui a au total 4000 étudiants dont 750 à 900 (150 annuellement) effectuent à la faculté de sylviculture 5 ians et demi d'études coupées de stages payés dans des services forestiers ou des stations de recherches.

L'enseignement par correspondance est également dispensé par l'Académie à cles étudiants aussi nombreux que les étudiants ordinaires.

Les enseignants sont formés grâce à l'aspiranture: les aspirants recrutés par concours parmi les diplômés d'études supérieures font 2 ans de pratique, puis en 3 ans préparent un " diplôme ) qui leur permet d'être agrégés des Sciences. Le doctorat est obtenu plus tard et plus difficilement par une thèse qui constitue un travail personnel important et original: les professeurs titulaires de chaire doivent en principe etre ( docteurs ).

Ils peuvent être élus membres de l'Académie des Sciences Agricoles, corps de savants très respecté jouant un grand rôle dans l'organisation de l'enseignement et de la recherche.

Les Instituts de Recherches sont également un débouché pour les Agrégés des Sciences et les docteurs, et ceux-ci peuvent devenir membre des Académie des Sciences Agricoles s'ils ont acquis une certaine notoriété.

Pour l'Ukraine, la recherche forestière est assurée depuis 1930 sous l'autorité de l'Académie des Sciences Agricoles de la République et avec une coordination à l'échelon national par l'Institut Ukrainien de recherches scientifiques de sylviculture et. de restauration des sols situé à Kharkov et comprenant 16 divisions et laboratoires:

1 - Sylviculture.

2 - Hydrologie forestière.

3 - Typologie.

4 - Reboisements.

5 - Production des graines et pépinières.

6 - Protection contre insectes et maladies.

7 - Amélioration des essences forestières.

8 - Bandes protectrices.

9 - Lutte contre l'érosion.

10 - Reboisement des rives des réservoirs.

11 - Reboisement des dunes.

12 - Laboratoire de physiologie.

13 - Laboratoire de pédologie. 
14 - Mécanisation des travaux.

15 - Laboratoire de photographie.

16 - Organisation et Méthodes.

Il dispose en outre d'un réseau complet de stations d'essais régionales auxquelles sont parfois annexés des (" points d'appui ) moins importants: chacune des stations a une activité spécialisée liée à la région. Elles sont au nombre d'une douzaine réparties dans les diverses zones climatiques depuis les forêts de montagne des Carpathes jusqu'à la Crimée méditerranéenne en passant par la steppe sans arbre où les problèmes de protection contre l'érosion sont très importants.

Le personnel comprend au total (pour un pays comparable à la France) près de 500 personnes :

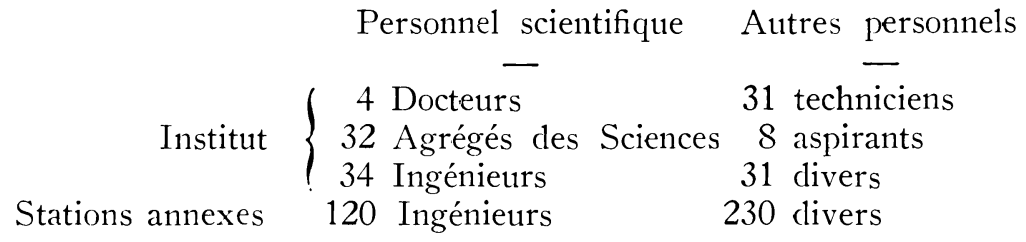

Nous avons visité à Kharkov l'Institut modestement installé et avons passé 3 jours dans la retraite campagnarde de sa Station régionale de Trostianetskaya consacrée surtout à l'étude très poussée, et parfois plus théorique que pratique, des reboisements en chêne pédonculé et pin sylvestre associés à des essences variées. Les expériences sont menées, avec l'aide du personnel local pour l'exćcution seulcucut dans une Inspection (Leskhoz) comprenant quelque 28000 ha autour de la station.

\section{La Foresterie dans les régions visitées}

Elle a pour le forestier français des aspects assez curieux et cela tient au tracé de notre voyage: sur le milliard d'hectares de forêts de l'U.R.S.S., 10 millions seulement se trouvent en Ukraine et en Georgie où nous avons passé 23 des 33 jours de notre séjour en U.R.S.S.

Nous n'avons fait qu'effleurer à Léningrad la zone forestière ou taïga et c'est clans la visite au Leskhoz Siverski que nous avons retrouvé des problèmes comparables aux nôtres: ailleurs, nous avons visité des plantations expérimentales, vu des forêts-parcs et d'autres qui allaient être mises en réserve ou transformées pour devenir " (les espaces verts ». Parfois même ceux-ci étaient créés de toutes pièces par les forestiers dont c'était le principal rôle. Ainsi l'U.R.S.S. a fait deux parts de ses forêts : celles consacrées à 


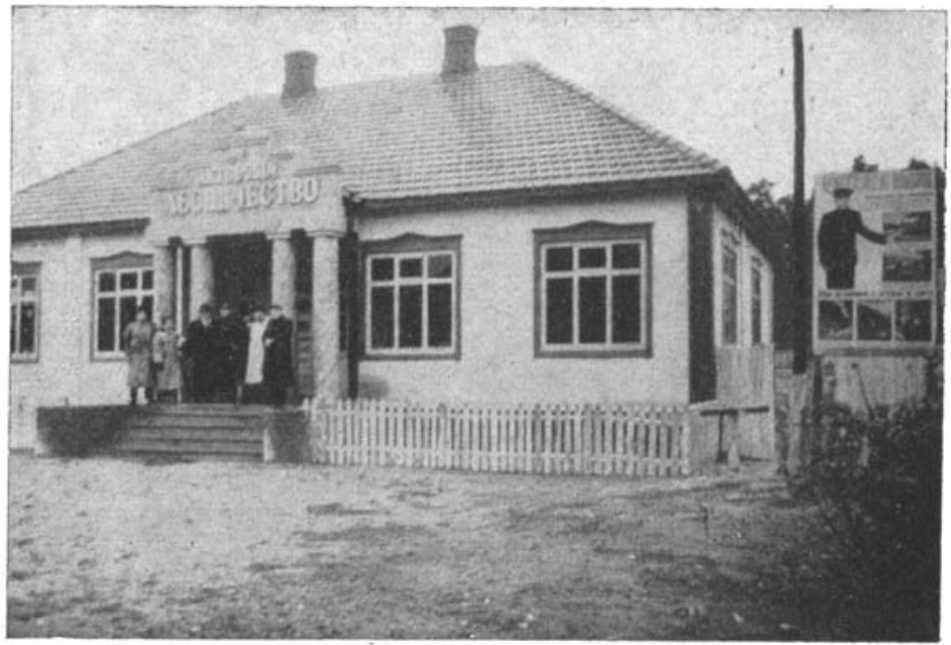

1 - Le bureau d'un cantonnement en Ukraine.

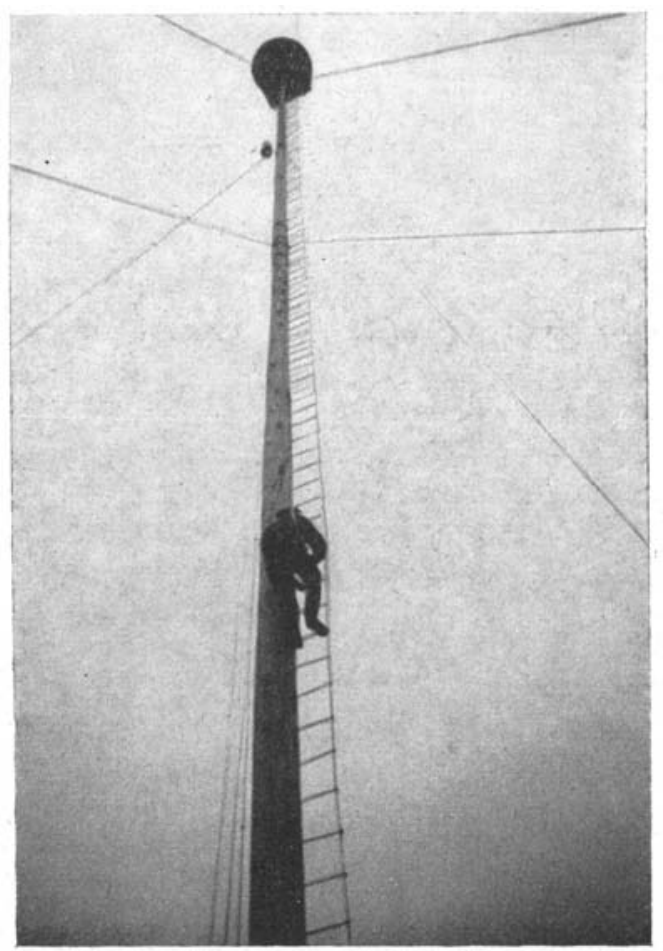

2 - Pylone de surveillance contre l'incendie, région de Léningrad. (Clichés Pourtet.) 
la production dans les régions peu peuplées, les autres essentiellement réservées à l'agrément et à la protection partout où la population est dense.

Nous nous bornerons donc à décrire sommairement les types de forêts et les essences vus dans les régions visitées, les travaux qui y étaient faits, l'orientation qui leur était donnée en insistant sur les points qui nous paraissent pouvoir intéresser le lecteur français et lui être utiles.

$1^{\circ}$ Région de Léningrad: C'est le Sud de la zone résineuse et le Mélèze qui règne au Nord et à l'Est n'est plus spontané; pins sylvestres de type (" Riga ), bouleaux, épicéas et trembles composent la forêt, la dernière espèce étant la moins recherchée. Les arbres sont élancés et généralement minces, la forêt est traitée en futaie régénérée naturellement avec une révolution variant de 80 à 130 ans suivant la qualité des sols. Ceux-ci, d'origine glaciaire, sont généralement mouilleux sans pente sensible et il faut un nivellement précis pour réaliser le réseau de drainage indispensable à la bonne santé de la forêt et même à sa production. On estime que l'assainissement peut augmenter celle-ci de 2 à $5 \mathrm{~m}^{3}$ par ha et par an. Ce résultat spectaculaire et qui mérite d'être retenu est obtenu avec des collecteurs primaires d'un mètre de profondeur réalisés à la pelle mécanique et des secondaires de $0,70 \mathrm{~m}$ effectués avec une charrue fossoyeuse. Au total, il y a 60 mètres cle fossés à l'hectare, ils sont curés tous les 10 ans par les mêmes moyens mécaniques: les tracteurs à chenilles, de 90-100 CV, utilisés semblent avoir une excellente adhérence sur ces terrains mouilleux. Deux autres problèmes préoccupent les forestiers de cette forêt relativement nordique - les incendies, qu'un bon équipement permet de déceler et cle combattre efficacement: les pylones de surveillance sont constitués par un simple mât muni d'échelons sommaires, mais l'ascension comme la descente du guetteur sont rendues très aisées et surtout rapides par la présence d'un câble auquel il s'attache et qu'un contrepoicls entraîne - la lutte contre l'aune blanc envahissant et gênant les régénérations naturelles des conifères: elle est assurée avec un plein succès par des phytocides classiques épandus par avion lorsque la surface à traiter dépasse 10 hectares.

Après les coupes, la régénération, aussi bien naturelle qu’artificielle, est également parfois entravée par l'engazonnement du sol, l'Institut de recherches forestières de Léningrad a conçu un appareil très original " la déchireuse de gazon en ancre ») qui doit réaliser un bon crochetage malgré les obstacles divers. Nous ne l'avons malheureusement pas vue à l'nuvre, mais nous avons pu la photographier à l'Exposition permanente des réalisations de l'économie nationale de Moscou. 


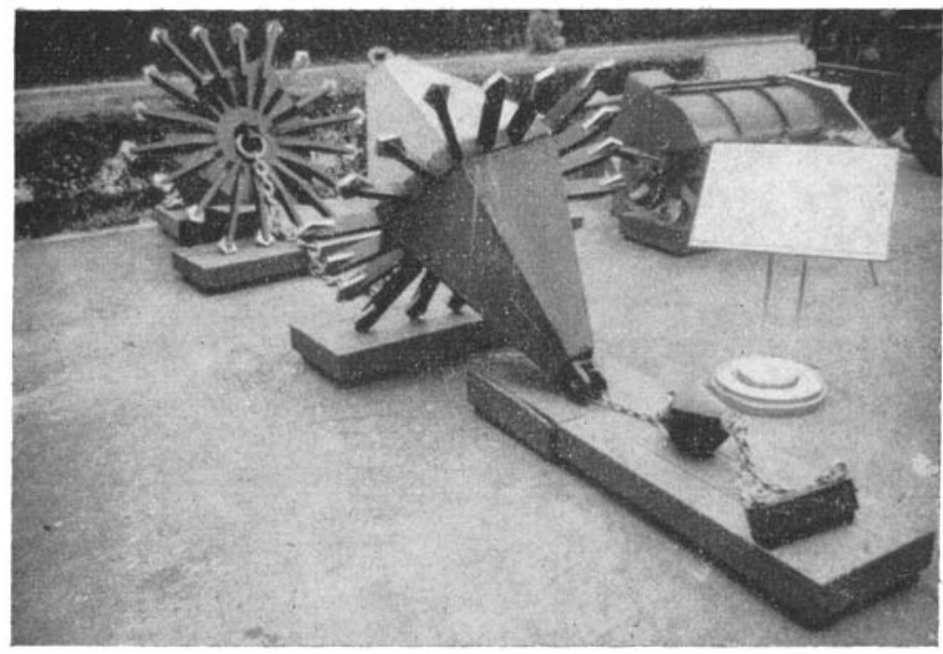

3 - Déchireuse de gazon en ancre.

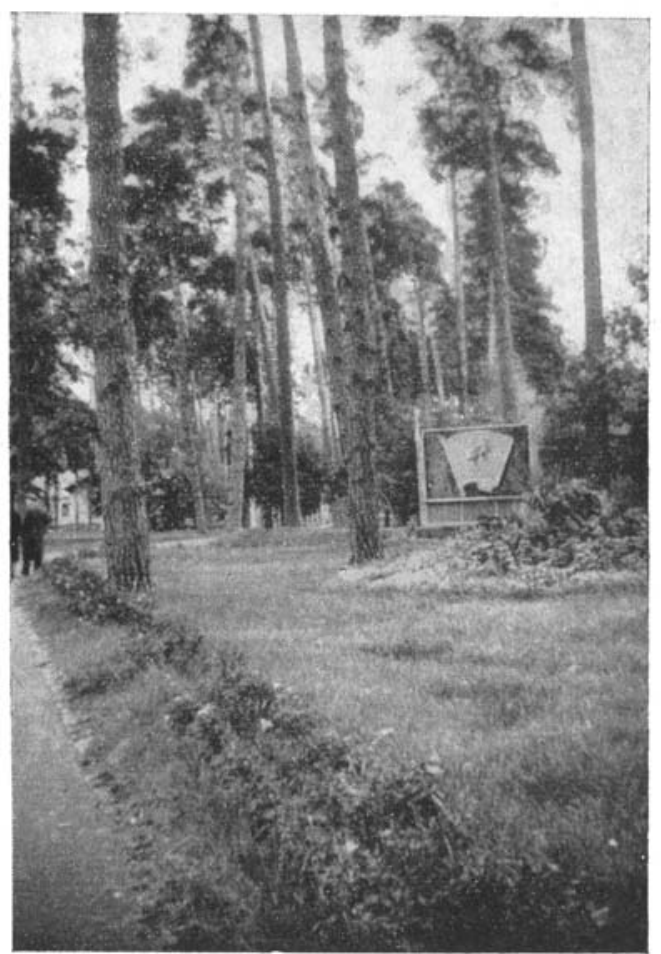

4 - Camp de pionniers aménagé sous une futaie de pins sylvestres près de Kiev.

(Clichés Pourtet.) 
$2^{\circ}$ Ukraine Nord-Occidentale: Ici, deux essences se partagent les forêts spontanées: le pin sylvestre et surtout le chêne pédonculé, celui-ci est essentiellement l'arbre ukrainien: sur les excellents sols de tchernozem, il peut vivre et même ètre extraordinairement vigoureux sous un climat relativement sec. Les révolutions sont courtes, 120 ans, avec une circonférence de $120 \mathrm{~cm}$ : cependant, dans l'Ouest, près de Jitomir, où Honoré de Balzac tenta d'exploiter des chênes pour les importer en France, on trouve des bois de belle qualité et de forte dimension réservés sous le règne de Pierre le Grand pour la marine.

Le pin sylvestre, généralement accompagné de bouleaux est dominant sur les sables. Chêne et pin sylvestre sont très généralement régénérés artificiellement. Cela a parfois amené pour le second de fâcheuses introductions de graines qui ont donné des arbres contrastant avec la rectitude satisfaisante des peuplements les plus fréquents et que leurs courbures et leur manque de verticalité font qualifier localement de " Pins ivres ).

La révolution généralement adoptée pour le pin sylvestre est 100 ans.

Les techniques de régénération artificielle font toujours appel à un travail du sol parfois réalisé mécaniquement avec des outils comparables aux nôtres, mais généralement la main-cl'œuvre rurale permet le travail en plein par l'utilisation très large de la méthode économique des concessions de culture accordées pour 3 ans. La culture des plantes sarclées et particulièrement des pommes de terre permet ainsi d'assurer l'entretien des jeunes plants de façon parfaite et gratuite.

Le reboisement est exécuté par semis pour le chêne, par plantation de semis d'un an pour les autres essences: l'écartement des lignes est assez grand $-2 \mathrm{~m}$ en moyenne - tandis que l'espacement sur les lignes est très réduit $-0,50 \mathrm{~m}-$, la densité est donc très forte, mais le plus original à notre avis est l'effort minutieux pour obtenir un peuplement mélangé de composition satisfaisante et des expériences multiples visent à trouver les associations qui conviennent le mieux. L'Académicien Lyssenko recommande le mélange de chêne et cl'érable plane et nous avons vu des peuplements très satisfaisants ainsi constitués; au contraire, le mélange du chêne ct de l'épicéa est consicléré comme néfaste pour le second d'ailleurs médiocre en Ukraine Centrale, mais l'association du chêne et du pin sylvestre est consiclérée comme bénéfique pour les cleux essences; le mélèze aurait une influence positive sur le frêne et négative sur le chêne; le tilleul augmenterait la quantité de racines nutritives des pins, etc... Enfin, les modalités de ces mélanges sont méticuleusement étudiées: pied par pied ou par ligne ou groupe cle lignes, en chaînons, en clamiers... : tous dispositifs difficiles à réaliser sur 
de vastes chantiers et que seule l'abondance de la main-d'œuvre permet tout au moins théoriquement.

Dans les peuplements obtenus et pour cette même raison, les coupes cl'amélioration commencent tôt: dans les 10 premières années, on fait 2 ou 3 nettoiements avec assez souvent l'élagage des jeunes résineux, les produits de ces opérations trouvent sans peine des utilisations. A l'âge cle 10 ans commencent des éclaircies généralement d'intensité moyenne $(15 \%)$ dont la rotation est d'abord de 5 ans puis de 10 ans.

Bien souvent, comme nous l'avons dit, le but cle ces peuplements n'est qu'accessoirement la production et clans un vaste périmètre autour des grandes villes, ils sont orientés vers la constitution de forêts-parcs d'agrément, gérées par les services forestiers dépendant des municipalités.

C'est ainsi que Kiev, ville d'un million d'habitants, dispose de près de 1450 hectares de parcs, squares, massifs floraux et alignements dans la cité même, mais aussi dans un rayon de $25 \mathrm{~km}$, de 39000 ha de forêts bien percées et entretenues: il s'agit en particulier de futaies âgées de pin sylvestre, avec un vigoureux sousétage naturel de chêne pédonculé. Parfois même, des camps de vacances y sont installés avec gazon et fleurs sous les grands arbres.

Deux constatations serviront de conclusion à ces quelques mots sur la sylviculture ukrainienne: la réussite pratiquement totale et constante des reboisements, nouvelle et éclatante démonstration de l'intérêt du travail du sol, - le remarquable comportement du clêne pédonculé qui pendant les 10 ou 20 premières années a une croissance égale à celle du pin sylvestre; nous avons vu des chênes semés en 1948 et dont la circonférence à 1,30 m était en 1959 de 25 à $35 \mathrm{~cm}$ avec une hauteur de 6 à $8 \mathrm{~m}$. Ensuite les éclaircies arrivent aisément à protéger le chêne contre le résineux.

$3^{\circ}$ Les régions méridionales - Crimée et Georgie: L'une et l'autre ont un climat subtropical, de type méditerranéen à proximité immédiate de la mer, plus aride et steppique à l'intérieur; leur topographie est accidentée, les montagnes s'élèvent brusquement depuis la côte et on passe très vite de la végétation la plus méridionale aux forêts mélangées d'altitude, elles atteignent la crête en Crimée (1500 m environ) et s'arrêtent à $2500 \mathrm{~m}$ sur les flancs sud du Caucase.

Ces forêts étaient particulièrement intéressantes pour nous et nous consacrerons un paragraphe spécial au sapin de Nordmann et à la sylviculture en Georgie. Cependant, en Crimée, sur les pentes abruptes en amphithéâtre dominant Yalta, une essence a retenu notre attention, c'est le pin de Tauride (Pinus laricio var. taurica $=P$. nigra var. pallasiana): il occupe une aire bien isolée de l'aire balkanique et asiatique des variétés orientales de pins 
laricios et s'y est maintenu lors des glaciations en raison de conditions climatiques favorables; formant un massif de 7000 ha, il s'étage de $200 \mathrm{~m}$ d'altitude à $1100 \mathrm{~m}$ où il se mélange au pin sylvestre et au hêtre avant de faire place à ce dernier (Fagus orientalis). C'est un bel arbre, assez trapu, pouvant atteindre de fortes. dimensions et qui se régénère naturellement avec vigueur. Déjà introduit áux Barres où il a une forte production (1), il peut être intéressant pour nous, car aussi méridional que le pin de Salzmann, il a une forme meilleure.

Les forestiers de Crimée s'y intéressent et l'utilisent dans les plantations de conservation des sols réalisées avec de puissants moyens mécaniques au-dlessus de la Mer Noire (bandes travaillées, terrasses de niveau ouvertes à l'angle-dozer sur une pente de $70 \%$ ).

Mais, ici plus qu'ailleurs l'importance du tourisme, le développement des maisons de repos et de soins donnent au forestier comme rôle essentiel la " foresterie touristique ». En Crimée l'Arboretum Nikitski, un des premiers du monde, dont les collections admirablement situées ont été commencées il y a plus de 140 ans et sont remarquablement soignées, fournit une riche gamme d'essences pour l'aménagement des parcs et des abords des villes. Un vaste projet vise même à enrésiner essentiellement avec le Pin de Tauride plus de 180000 hectares de taillis chêne-charme dans un but sanitaire, le pin étant consicléré comme bactéricide!

Au Caucase, c'est un autre pin existant déjà en Crimée, qui joue ce rôle: Pimus hamata $=P$. sosnoz'skii, arbre de belle forme, très voisin du pin sylvestre, dont Gaussen l'a cependant distingué, il est planté à proximité des stations thermales même dans les clairières des forêts de sapin et l'épicéa.

Aux abords les villes, le travail est infiniment plus ardu que dans la plaine d'Lkraine, mais même dans l'intérieur sur les pentes sévèrement dénulées et érodées qui entourent Tbilisi, les forostiers ont entrepris un énorme travail: petites banquettes de niveau faites à la main pour recevoir de jeunes plants, énormes trous creusés parfois mécaniquement oì l'on installe cle véritables arbres transportés avec leur motte, entretien manuel, arrosage par un réseau cle canalisations métalliques. Grâce à cet effort, cles milliers d'hectares se couvrent de verdure et on peut espérer que la capitale de la Georgie aura dans quelques années une remarquable ceinture verte. A côté (les pins dont nous venons de parler et de quelques feuillus et arbustes (Rhus cotinus joute un grand rôle là comme en Crimée), les (leux principales espèces utilisées sont une forme de Pinus brutia, P. cldarica, autochtone en Georgie, et, dans les sta-

(1) J. Pardé et P. Turpin. - Caractéristiques et production des peuplements de pin laricio du domaine des Barres. Rev. for. fr., mai 1959, p. 376391 . 


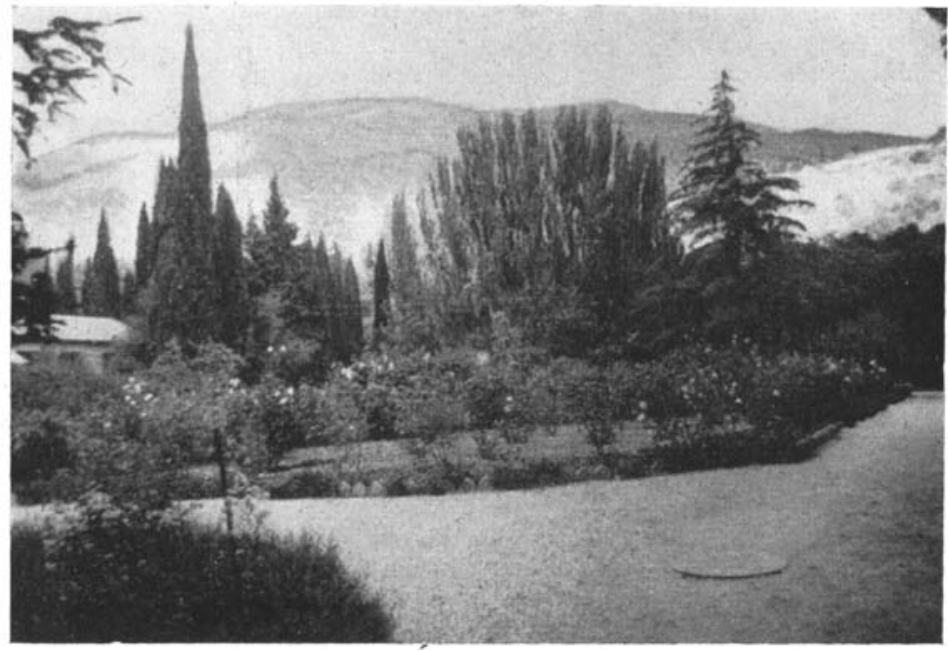

L'arboretum Nikitski et les monts de Crimée.

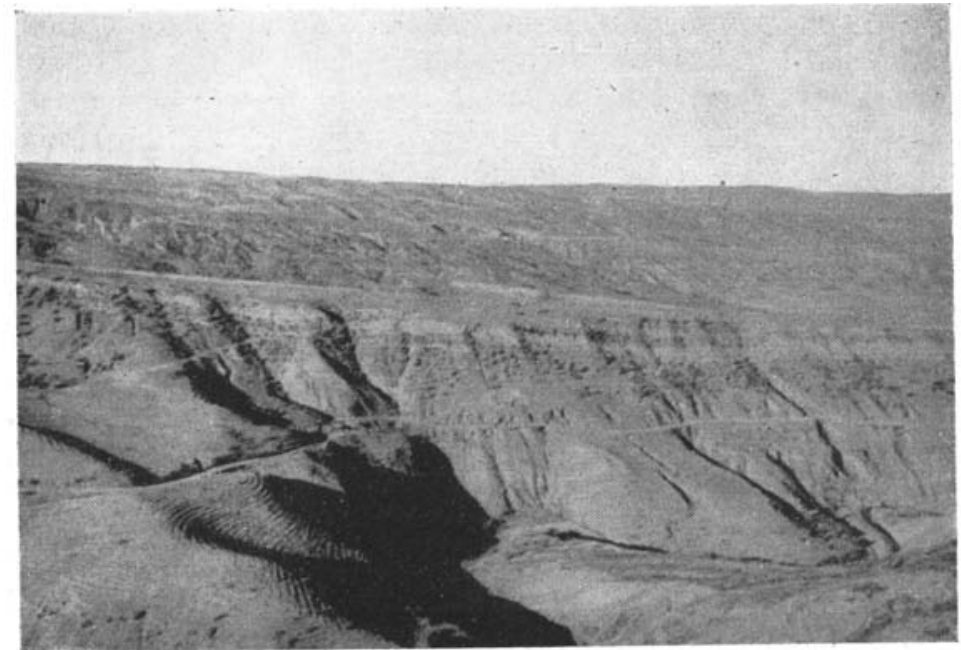

Les pentes déboisées et érodées des environs de Tbilisi: Travaux de restauration.

(ClichésPourtet.) 
tions pluis favorables, Cedrus deodara. Le Cèdre de l'Himalaya réussit admirablement clans toute la Georgie et sur le pourtour de la Mer Noire: il y fait prẹve d'étonnantes plasticité et résistance à la sécheresse. C'est à Tbilisi et aux environs un magnifique arbre d'avenue alors que la pluviosité annuelle n'atteint pas $500 \mathrm{~mm}$. Plus classique pour nous, le cyprès méditerranéen et le thuya de Chine (Biota oriontalis) sont également largement utilisés ainsi que les peupliers développés depuis peu.

\section{Le Sapin de Nordmann}

Abies nordmanniana est le seul sapin spontané dans la partie de l'U.R.S.S. que nous avons visitée et il n'y existe qu'au Caucase occidental et dans les chaines secondaires qui s'y rattachent et se prolongent vers le sud jusqu'en' Turquie. Nous en avons vu deux stations.

a) En remontant en ( Gas », la Jeep soviétique, par un chemin forestier difficile, le cours de la rivière Niedzoura, qui donne son nom à la première forêt visitée, nous avons vu apparaître le sapin de Nordmann, sur le versant nord, à partir de $800 \mathrm{~m}$, et sur le versant sud, à partir de $1000 \mathrm{~m}$. L'épicéa d'Orient l'accompagne partout. Dans le bas de l'aire, le charme du Caucase (forme peu différente de $C$. betulus) leur est associé en faible proportion; plus haut, il est remplacé par le hêtre d'Orient. C'est à $1200 \mathrm{~m}$ que dans cette forêt le sapin de Nordmann paraît trouver son optimum : la pluviosité est de lordre de 1000-1200 mm, et le sol est un sol brun, sur roches métamorphiques ou volcaniques (gneiss, anclésite, basalte). I.e sous-bois est constitué principalement de Houx et d'Acer lactum. Dans la flore herbacée, on trouve entre autres Festuca montana et une gentiane voisine de Gentiana ciliata (qui existe aussi sur le plateau de Langres). Le peuplement, traité en futaie jardinée, comprend, en proportions sensiblement égales, du sapin de Nordmann et de l'épicéa d'Orient; il n'y a pratiquement pas de feuillu, et l'aspect de la forêt est très semblable à celui de la forêt vosgienne.

Les arbres sont beaux, bien élagués, longs (30 à 32 mètres) et vigoureux. Les plus gros sapins, dans des zones forestières non exploitées, atteignent, nous dit-on, 50 à $60 \mathrm{~m}$ de hauteur.

Le jardinage est pratiqué pied par pied lorsque la pente dépasse $10 \%$, par bouquets dans le cas contraire. La régénération est entièrement naturelle et s'obtient sans difficulté.

L'épicéa fournit un bois excellent, nettement plus apprécié que celui du sapin qui est plus tendre. L'épicéa est donc favorisé par le forestier, mais l'écologie des deux essences est très voisine, et il n'est guère possible d'orienter les régénérations en faveur de l'une ou de l'autre. 
Sur 1200 hectares, une technique un peu particulière a été appliquée:

- En 1935, on a exploité tous les bois ayant plus de 1,50 m de circonférence. Les arbres de cette grosseur se trouvant par bouquets de 3 à 5 pieds, on a ainsi ouvert des trouées de 5 à 10 ares. Le volume réalisé a été de $100 \mathrm{~m}^{3}$ par hectare. Une régénération abondlante, à dominante de sapin, s'est développée dans les trouées.

En 1958, une seconde coupe a réalisé tous les bois de plus de 1 mètre de tour (soit 60 à $65 \mathrm{~m}^{3} / \mathrm{ha}$ ), ce qui a élargi les trouées précédentes et en a ouvert de nouvelles.

- Il est prévu dans les 20 années à venir, de réaliser en une ou deux fois, tout le reste du vieux peuplement (120 à $150 \mathrm{~m}^{3} / \mathrm{ha}$ ). Le résultat final attendu est un peuplement par bouquets, dans lequel l'écart extrême des âges ne dépassera guère 40 ans, et qui se rapprochera donc de la régularité.

I1 est intéressant de signaler que les graines de sapin de Nordmann importées en France par la maison Vilmorin en 1957, et utilisées dans beaucoup de pépinières du Fonds forestier national, ont été récoltées dans ces peuplements: nous avons été heureux de connaitre les vrais parents de nos enfants d'adoption, et de les voir aussi beaux.

b) Une route extrêmement pittoresque et souvent grandiose, remontant les gorges de la rivière Bzibi, conduit en $50 \mathrm{~km}$ de Gagra au bord de la Mer Noire, jusqu'au lac Ritza, lac de barrage naturel, à $950 \mathrm{~m}$ d'altitude. C'est à partir de $600 \mathrm{~m}$ qu'on voit apparaître, presque simultanément, l'épicéa d'Orient et le sapin de Nordmann, d'abord à l'état isolé, au milieu des taillis de charme d'Orient (Carpinus orientalis), puis par bouquets de plus en plus étendus. A ce niveau, leur végétation est médiocre, leur forme généralement défectueuse; les bouquets sont clairs et il existe un sous-étage très important de grands buis (Buxus colchica) pouvant mesurer $30 \mathrm{~cm}$ de diamètre. En montant, ce sous-étage se transforme: le buis disparaît, il est remplacé par le laurier-cerise et par un rhododendron ; en même temps, la forme des arbres s'améliore.

Au niveau du lac Ritza, le climat malgré l'altitude, est assez chaud (extrêmes thermiques absolus: $+35^{\circ}$, $-14^{\circ}$; minima courants: -4 à $-6^{\circ}$ ). Néanmoins, le lac est gelé en hiver, et les chutes de neige sont abondantes, pouvant atteindre 5 mètres. La pluviosité est élevée: $2000 \mathrm{~mm}$ par an. Les sols sont variés, mais à dominante calcaire. Ils donnent naissance à des sols bruns forestiers, avec humus neutre ou faiblement acide.

La forêt résineuse s'étend jusqu'à $1800 \mathrm{~m}$ d'altitude. Le sapin de Nordmann paraît trouver ses meilleures conditions de végétation vers 1300-1400 m. A ce niveau, le sous-bois est très réduit, mais il $\mathrm{y}$ a, en sous-étage, du hêtre et de l'érable Sycomore; dans l'étage 
dominant, la proportion d'épicéa est très faible: le sapin de Nord- . mann est presque pur. Nous avons vu des individus remarquables par leur forme (fûts cylindriques et bien élagués) et leurs dimensions ( $4 \mathrm{~m}$ de tour sur $38 \mathrm{~m}$ de hauteur). Les arbres pourtant âgés ne forment pas la table et ont une silhouette relativement étroite. Les forestiers locaux nous ont indiqué que, sur les sols de pre-

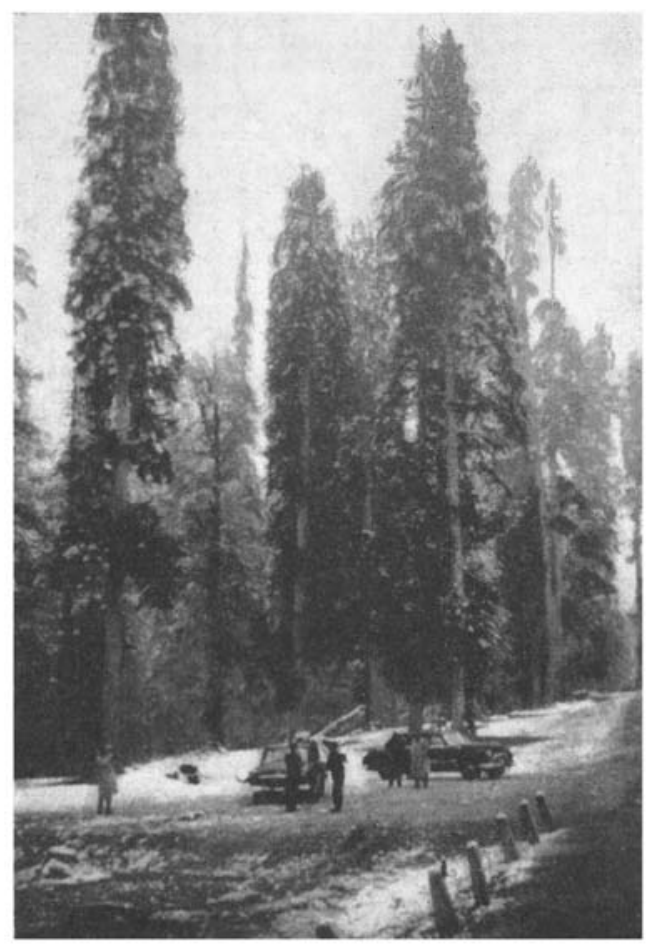

Abics Nordmanniana dans la vallée du lac Ritza sur le versant sud du Caucase (1300 m d'altitude)

(Cliché Pourtet.)

mière qualité, on trouve beaucoup de sujets dépassant 2 mètres de diamètre et 55 mètres de haut. L'âge d'exploitabilité normale est fixé à 120 ans, ce qui correspond à une circonférence de 130 à 140 centimètres. Les peuplements sont traités par la méthode du jardinage: soit pied à pied sur les sols superficiels où les peuplements sont relativement clairs et où la régénération est permanente - soit par trouées sur les sols profonds. La régénération naturelle est contrariée, dans toute l'aire du sapin - mais moins gravement, semble-t-il, dans la zone médiane - par la concurrence des morts- 
bois du sous-étage (Rhododendron, Laurier-Cerise). Les forestiers préfèrent l'épicéa, qui donne des bois de haute qualité (bois de résonance), mais les jeunes sapins poussent plus vite que les jeunes épicéas.

La croissance du sapin de Nordmann est soutenue jusqu'à 200 ans et plus. L'accroissement de ses peuplements est habituellement, dans les conditions locales, de 5 à $10 \mathrm{~m}^{3}$ par hectare et par an.

Les plus beaux peuplements ont un volume sur pied de 1300 à $1400 \mathrm{~m}^{3}$ par hectare à 130 ans.

Le sapin de Nordmann n'est pas utilisé en plantations par les forestiers soviétiques.

\section{L'aménagement des bandes-abris}

Dans toutes les régions du. monde et même dans les pays tempérés comme le nôtre, l'intérêt de la protection contre le vent par l'implantation de ricleaux d'arbres se révèle chaque jour plus nettement. Ce problème a une importance capitale dans les pays comportant de vastes étenflues plates et relativement arides; c'est pourquoi la Russie fut à l'origine des études et des techniques sur cette question. Les savants russes Dogoutchaiev et Williams, agronomes et surtout pédologues, ont mis au point les méthodes qui avaient vu le jour dans la steppe Kamennaia (au nord-est de Kharkov) vers 1860: " c'est (là) que la grandle utilité des bandes forestières pour la protection des champs a été cémontrée de façon la plus probante. De larges bandes d'arbres avaient barré la route aux vents secs et ont permis de doubler la récolte de blé )) (1).

Plusieurs missions étaient venues en Russie avant la nôtre, étudier cette question, mais notre but était de l'examiner dans l'optique des forestiers français, gênés pour appliquer les principes classiques des bandes-abris. par la structure agraire et les dimensions modestes de notre pays. Nous avions donc l'impression que peu de choses seraient transposables de l'U.R.S.S. en Europe Occidentale: les premiers renseignements impressionnants, recueillis à l'Exposition de Moscou confirmaient cette idée préconçue. D’immenses “ bandes d'état ) de 40 mètres de large coupant les vastes plaines - $1200 \mathrm{~km}$ pour la bande Bielgorod-Don que nous avons vue aux environs de Kharkov - et d'autres bandes plus modestes mais généralement de plus de 10 mètres de large, compartimentant les cultures et formant un réseau dont la densité idéale varie de 3 à $4 \mathrm{~km}$ pour 100 ha (compartiments de 100 à $200 \mathrm{ha}$ ) à 5 à $8 \mathrm{~km}$ pour la même surface (compartiments de 300-500 mètres sur 1000-2000 mètres).

Les modalités de réalisation étaient aussi minutieusement com-

(1) N. Baranski. - Géographie économique de l'U.R.S.S. Edition française. Moscou, 1956, p. 128. 
pliquées que les reboisements expérimentaux de l'Ukraine. Les schémas suivants en donneront une idée:

a) Bande d'état - type spécial pour abords d'agglomération (plus décoratif):

19 lignes à $1,50 \mathrm{~m}$ les unes des autres $-39 \mathrm{~m}$ au total $\mathrm{y}$ compris les bordures.

Lignes $n^{0} 1$ et 19: arbres fruitiers et arbustes divers en alternance.

Ligne $n^{\circ} 2,3,4,5$ : séries de 8 plants comprenant chêne pédonculé, arbustes divers, pin sylvestre alternant avec des séries du même nombre, composées de Pin sylvestre, sureau rouge et sorbier des oiseleurs.

Lignes $n^{\circ} 7,8,9$ : alternance des premières séries précédentes avec des séries bouleau, noisetier, Juglans mandshurica ou Phellodendron amurense.

Ligne $n^{\circ} 10$ : chêne pédonculé pur.

Lignes $n^{\circ} 11,12,13,14$ : alternance des premières séries précédentes avec des séries: Cornouiller, chêne rouge, épicéa.

Lignes $\mathrm{n}^{\circ} 15,16,17$ et 18 : alternance des premières séries précédlentes avec des séries troëne, marronnier et mélèze.

Même avec les entorses données dans la pratique au dispositif théorique, la réalisation est délicate si le résultat esthétique est satisfaisant!

b) Bande-abri à l'Institut de culture des plantes et de sélection prìs de Kharkov:

11 lignes à $1,50 \mathrm{~m}$, largeur totale $16 \mathrm{~m}$ avec les bordures.

Lignes impaires: Caragana arborescens.

Lignes $n^{\circ} 2$, 6 et 10: chêne péclonculé alternant avec érable plane.

Lignes $11^{\circ} 4$ et 8 : Fraximus viridis.

Au lieu d'être installés par individus isolés, les chênes sont semés en " nids » comprenant 45 glands répartis suivant un schéma précis, conformément à la théorie de Lyssenko qui affirme que des sujets de la même espèce ne se nuisent pas, mais s'aident mutuellement par soudures des racines. Cette théorie interdit les éclaircies dans les groupes obtenus, mais elle n'est plus intégralement respectée.

Dans ces deux cas comme dans tous les autres exemples rencontrés, le sol était cultivé comme les champs voisins et entretenu aussi longtemps qu'il était possible: la réussite était généralement bonne et le développement assez rapide, mais il apparaissait de manière évidente que la production possible de bois n'était pas envisagée.

Cependant, malgré l'effet bénéfique incontestable des rideaux, on nous signalait des difficultés avec les agriculteurs et en particulier 
les conducteurs de tracteurs, tandis que l'utilité des arbustes destinés à " fermer les rileaux » était contestée ainsi que certaines théories sur les mélanges.

$\mathrm{Ce}_{\mathrm{s}}$ indications précieuses étaient l'origine ou le signe d'une profonde transformation en cours dans la théorie des rideaux-abris: elle nous a été exposée à Odessa devant un exemple typique de simplification dans le choix des essences et à l'Acadénie forestière de Léningrad.

La conception des nouvelles bandes-abris soviétiques scmble devoir obéir aux principes suivants:

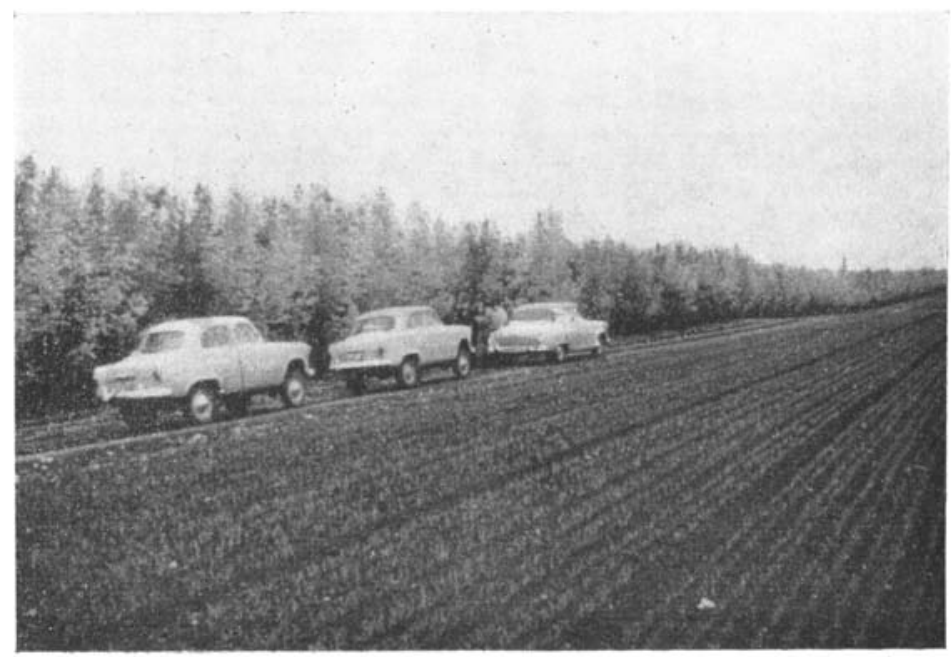

Bande-abri en Ukraine.

(Cliché Pourtet.)

- Il ne faut pas chercher à constituer un rideau opaque mais plutôt une " passoire ». Le " mur " présente des inconvénients lors de l'accumulation de neige; la " passoire 》 suffit pour éviter les méfaits du vent. Les arbustes destinés à garnir la base de la bande n'ont donc pas d'intérêt et on renoncera à les introduire.

- La largeur de la bande n'a pas une grosse importance et on estime que deux lignes pourraient suffire.

- L'écartement entre les lignes n'influe pas considérablement non plus: le critère essentiel est la possibilité de travail du sol pour faciliter l'installation de plants qui par définition doivent affronter la dessiccation causée par le vent. Les bandes étaient toujours implantées en conditions agricoles, il est facile d'assurer leur entretien avec le matériel de culture et l'écartement des lignes est dicté par la 
voie des tracteurs. On estime ainsi dans la région d'Odessa que $3 \mathrm{~m}$ est un bon chiffre.

- On doit recourir à des essences adaptées aux conditions locales et supportant en particulier le vent. Cette raison, ajoutée au souci de concurrencer le moins possible les cultures riveraines conduit à retenir des essences pivotantes.

Le premier rôle de la bande étant de jouer sans interruption et le plus longtemps possible son rôle d'abri, on recourra à des essences longévives susceptibles d'être régénérées par recépage sans pour cela abandonner toute idée de production économique si cette dernière reste compatible avec la fonction de la bande. Cependant, il existe généralement pour une station donnée une ou plusieurs essences à croissance plus rapide que celle des essences longévives destinées à constituer définitivement la bande; c'est souvent le cas des Peupliers. L'essence à croissance rapide sera associée à l'essence longévive, elle assurera plus précocement protection et revenu, mais ne jouera qu'un rôle transitoire.

Par exemple, clans les terres noires d'Ukraine, l'association chêne pédonculé - peuplier paraît convenir parfaitement à de tels objectifs.

Il est inutile d'insister sur l'intérêt de tels enseignements, aboutissement de 100 ans d'études, ils pourront nous guider pour une transposition française qui devient possible.

\section{Conclusions}

L'intérît manifesté à notre retour pour nos impressions d'U.R.S.S. par tous nos interlocuteurs français nous dispense de dire que ce voyage fut intéressant et par certains points passionnant. Du point de vuc technique, l'intérêt des contacts que nous avons pris, l'utilité des observations que nous avons pu recueillir et le bénéfice que nous tirerons de la connaissance cle certaines techniques qui nous ont été décrites notamment en ce qui concerne les bandesabris, sont incontestables.

Les précisions que nous avons obtenues sur le comportement de cortaines essences et en particulier sur l'écologie du sapin de Nordmann clont peu de forestiers français ont pu admirer les peuplements spontanés seront bénéfiques pour les reboiseurs.

Tout cela n'est pas négligeable, mais l'essentiel nous parait d'avoir fait la connaissance d'un pays différent du nôtre et d'avoir constaté que la foresterie reste un lien étroit entre ceux qui la servent quel que soit ce qui pourrait les séparer.

Préparé au ministère de l'Agriculture de l'U.R.S.S. par M. KARTCHENko pour le département de Collaboration Scientifique internationale et par M. Kavaline pour le département de Sylviculture, 
notre programme avait le souci de réaliser fidèlement les objectifs cle notre mission, tout en nous faisant voir dans des conditions aussi agréables que possible aussi bien des aspects touristiques parfois inattendus de ce pays inconnu de nous que les sujets techniques qui nous intéressaient.

Avec les grandes diversités que des régions échelonnées sur $3000 \mathrm{~km}$ peuvent présenter dans les habitudes et les modes de vie, les forestiers qui nous reçurent ou nous accompagnèrent s'ingénièrent à nous être agréables: nous n'oublierons pas l'hospitalité russe matérialisée par exemple par l'accueil de M. Loukanietz, sous-directeur du Service Forestier d'Ukraine sur le quai de la gare de KKiev à 4 heures du matin sous une pluie glacée, ou encore par la gentillesse de tous les instants de M. Tschodrichvili, Ministre des forêts et de la chasse de la république de Georgie, qui fut notre guide pendant plusieurs journées.

Notre fidèle accompagnateur, l'Aspirant (1) Baranov fut le clévouement personnifié s'ingéniant à faire disparaitre de notre vie tout souci matériel tandis que l'intelligence, l'aimable dévouement et la compétence technique de notre interprète, Mlle Anna IvanovNA étaient les éléments déterminants de la réussite de notre voyage.

(1) Voir ci-dessus, page 391).

Un voyage spécial

s'adressant aux Exploitants forestiers

et à ceux des Scieries Papeteries

Fabriques de contreplaqué

est prévu à l'occasion du $\mathrm{V}^{\mathrm{e}}$ congrès forestier mondial de Seattle S’adresser à MM. Riss et Cie, 68, avenue des Champs-Elysées - Paris $\left(8^{\mathrm{e}}\right)$. 